\title{
Situating Productive Play: Online Gaming Practices and Guanxi in China
}

\author{
Silvia Lindtner ${ }^{1}$, Scott Mainwaring ${ }^{2}$, Paul Dourish ${ }^{1}$, and Yang Wang ${ }^{1}$ \\ ${ }^{1}$ Department of Informatics, \\ University of California, Irvine, \\ Irvine, CA 92697-3440, USA \\ lindtner@ics.uci.edu, jpd@ics.uci.edu, yangwang@uci.edu \\ ${ }^{2}$ People and Practices Research, \\ Intel Research, 20720 NW Amberglen Ct, MS, \\ Beaverton, OR 97006, USA \\ scott.mainwaring@intel.com
}

\begin{abstract}
Economic activities in and around online gaming in China are often correlated in the West with practices of gold farming, or selling in-game currency to players for real money in online games. What can we learn about online gaming in China and about online gaming and online sociality more broadly when we look at economic and other "pragmatic" practices through which online gaming becomes meaningful to players? In this paper, we present findings from an ethnographic study of online gaming in China's urban Internet cafes to discuss implications for game design, and HCI design more broadly. Considering the ties between socio-economic practices, development of trust and culturally situated imaginings of self-hood and otherness, brings to the fore how online gaming in and of itself constitutes the means for practical achievements in day-to-day management of guanxi (social connection).
\end{abstract}

Keywords: Online game, China, productive play, serious gaming, guanxi.

\section{Introduction}

In an Internet café in Hangzhou, a city in the Eastern region of China and an hour train ride from Shanghai, we met Tao, a 27 year-old player of the online game World of Warcraft. We were sitting next to Tao informally conversing about the happenings on his screen, when he told us, while pointing to one of the virtual characters that kept re-appearing next to his own: I have a few close friends [like this] in the game. We have very good guanxi... I trust him and he trusts me... We all shared our phone numbers. We look after each others' [in-game] characters.

During a 6-week long ethnographic study on online gaming in China in 2007, we encountered many players like Tao who invoked guanxi when describing the various types of relationships they developed in online games like World of Warcraft and at the physical locations where game play took place. Guanxi is a Chinese construct of social relations and reciprocal exchange $[10,23]$. It is an important but complex frame in which certain social practices of material and emotional exchange are understood. 
Often, guanxi is practiced and experienced through both the flow of material gifts (or capital-as-gift) and favors and the build-up of emotional and moral values such as trust or resentment within a network of dyadic relationships [4]. How and to what extent guanxi in Chinese societies differs from more general and culturally widespread processes of social networking and social capitalism remains a contentious issue [6]. From the perspective of HCI, however, even if guanxi is no more than an exemplar of a more generic phenomenon, it provides a particularly useful and culturally specific vantage point from which to consider how the design of online games (and online social spaces more generally) can become implicated within larger practices of currying favor, building networks, managing reputation, and leveraging social capital.

In recent years the HCI community has become increasingly invested in online gaming research. For example, previous research efforts have drawn our attention to the affordances of online gaming technology for complex social dynamics such as the formation of stable groups over longer periods of time, management of and collaboration within large collectives of people, and facilitating social action and flexible game play $[2,5,15,17]$. More broadly, it has also been acknowledged that online sociality and play can have quite serious meanings for players and often impact players' lives and decision-making processes outside of the game [3, 5, 15, 17, 21] - especially in disruptive situations such as cheating in the game [21], shut down of game servers [17], break up of online groups [5], or when in-game activities take on the shape of work-like performances [22]. Edward Castronova, T.L. Taylor and Nick Yee, in particular, have highlighted how boundaries between play and work break down, how players make productive use of games (e.g. to earn money) or how gaming can also feel like work.

What happens, however, when playing the game is not just productive based on value generated within the game, but in fact fulfills pragmatic concerns beyond game play? Nowhere is this more evident, perhaps, than in the domain of online gaming in China. Though it is possible to approach this domain as a growing market, a cultural phenomenon, a state- and corporate-regulated infrastructure of hardware and software services, an online community, or even a challenging opportunity for user interface/experience design, it is more usefully considered a combination of all of these (and more). We argue in this paper for the efficacy of an approach that considers the contingency of the serious and playful [13] by applying it to an important theme that emerged from our ethnographic investigation of Chinese online gamers: how guanxi is shaping and being shaped by these gamers' perceptions, motivations, and behavior.

What we found in China were aspects of online gaming that render game play in and of itself a means for practical achievement, even when the online game is understood as exactly that: a game. We encountered gaming practices that were driven by pragmatic and socio-economic concerns such as maintaining and extending one's guanxi network, navigation around governmental restrictions of game play, and how to gain and/or maintain status and reputation in as well as outside of the game. Anthropologist Thomas Malaby insightfully points out that “...[games] are certainly, at times, productive of pleasure, but they can also be productive of many other emotional states"[13]. In line with Malaby's observations, an important aspect of understanding the complexities of online gaming in China required understanding the game's role within its wider material, social, and economic contexts. In previous work [11], we have provided an in-depth analysis of how game play in the Chinese Internet 
café spanned online and offline sites embedded in larger webs of political and socioeconomic structures. Building on this previous work, this paper expands concurrent notions of productive play $[5,17,24]$ in that we incorporate a wider range of serious practices including accomplishments within the larger society such as building one's career and social network (guanxi wang). The starting point of our analysis in this paper, thus, is to think through what "seriously" playing an online game such as World of Warcraft means not just in terms of instrumental activities in the game (that certainly also do have "serious" meanings beyond the game space), but also how the instrumental and serious are achieved and maintained through and around playing the game.

\subsection{Guanxi}

First, we wish to highlight that the work presented here acknowledges Chinese cultural particularities in relation to online gaming practices, while refraining from describing these practices as intrinsically Chinese $[6,10]$. Our goal was to examine the ways in which instrumentalism and sentiment come together in guanxi networks that are developed and/or maintained through online gaming practices in China, and not how guanxi practices we observed in and around online gaming in China might be similar or different from social capital in online games in the U.S. Emotional aspects of the material and instrumental exchanges that come together in guanxi are not easily visible to outside observers, and the combination of instrumentalism and sentiment thus often appears contradictory [6] and leads to associations with corruption and bribing. We have thus refrained from a direct comparison between the "here" and "there" and rather focused on the practical contingencies of entertainment and its situated uses in the cultural context of urban China. For the scope of this paper, we consider guanxi as a useful framework in its own terms, independent of how one might theorize its relationship to larger concepts such as individualism/collectivism. In our work, we followed the lead of scholarly experts in China studies $[$ e.g. 1, 10] in refraining from direct comparison between guanxi and understanding of individualism/collectivism in order to avoid an orientalist framing of "Chineseness."

Guanxi and its related socio-cultural constructs have been widely studied in anthropology, sociology, and business [1, 4, 6, 9, 10, 23]. For purposes of this paper, we schematize the key concepts as follows: First, guanxi, in its most basic form, is a social connection between two individuals over which gifts and favors (and, conversely, obligations for future reciprocation) flow [4, 10, 23]. Quite complex and farflung guanxiwang (guanxi networks) are assembled out of these dyadic links. A guanxi link may be strong or "quality" (trustworthy, mutual, or genuine) or weak (unreliable, coercive, or perfunctory). Second, guanxi is built upon a mutually recognized "guanxi base" or common ground. In common practice, a guanxi base may take the form of a kinship relation (a family tie), a shared birthplace, a shared school or workplace, a shared acquaintance with whom both people have guanxi, or shared playing of the same game [4]. Third, guanxi must be cultivated and maintained. Without active attention, guanxi may decay over time; preventing such decay requires token or substantive gift-giving and -receiving over extended periods of time.

What underlies these general features is a diverse range of guanxi: in addition to good or bad, or strong or weak, it can be commodified, political, or friendly [10]. In 
each of these cases, however, guanxi is understood in distinction from a particular way of acting in the world, a way based on subsuming one's interests to institutions and their rules and ideologies. Indeed, a line of scholarly research has interpreted guanxi as a form of "navigation around the system" [1]

The art of doing guanxi resembles a kind of game play, a skilled activity that is marked as social, not work, amateur not professional, personal not official [6]. And so, a-priori, one might expect guanxi to be quite compatible with online gaming: a place in which to make social connections, feel human closeness, and maintain friendships over time, with a distinct feeling of being apart from the "non-game" "official" "real life" world, however, deeply intertwined with one's everyday life. While some researchers speculated that economic changes might cause a decline of guanxi, recent research also shows that guanxi, instead of diminishing, has found new territory in which to evolve [23]. Online gaming, with its analogies to artful practice of guanxi, in this light, is a particularly fertile ground for guanxi's colonization and evolution. In this paper, then, we analyze how online gaming can provide exactly such a new territory for guanxi to be built and maintained.

\section{Method}

In the summer of 2007, we conducted ethnographic fieldwork in Beijing, Hangzhou, and Shanghai. Our main focus was the online game World of Warcraft (WoW), one of the most successful online games in China. In WoW, players create and develop an animated character in a setting derived from the fantasy game Dungeons and Dragons. Game activities include slaying monsters, fighting other players, and participating in a vibrant in-game economy. Although the game can be played alone, WoW is fundamentally a social game. We also encountered players of other popular games including Tencent's popular QQ Games, the Legend of Miracle 2 and With Your Destiny. Most fieldwork took place in physical sites of game play. We collected our data from observations, informal conversations, semi-structured interviews, and focus groups. Sometimes we asked our study participants to think aloud while they were playing or interviewed them during a game session. We conducted interviews in low- to highend Internet cafés (wang ba), in restaurants, and in workplaces, dorms, and homes. Usually, we interviewed players at their preferred game location or at a place nearby. Most interviews lasted about an hour although some were longer.

In the semi-structured interviews we questioned study participants about many dimensions of their gaming experience. We asked players how they got started playing, about their previous gaming experience, what they liked and disliked about WoW, whether they played with people they knew in real life, whether they had made friends online and in wang $b a$, whether they belong to a guild, and if so, the kinds of experiences they had in their guild. We discussed the use of game-related software extensions and websites, forums, and wikis external to the game. We asked gamespecific questions about character choice, naming of characters, game activities participants liked. We asked what players would change about the game if they could. We followed up conversational leads as they arose, consistent with standard ethnographic practice. We emphasize that this research was not intended to be a systematic study of the nature of guanxi around online gaming, rather guanxi emerged from the field work. 
Study participants were acquired through our own social networks and through serendipitous encounters in Internet cafes or places where game-related merchandises such as game magazines were sold, e.g. shopping malls or small street vendors. We interviewed 80 players, 56 male, with diverse backgrounds including students, young professionals, a factory worker, a middle school teacher, senior players in their 50's, a marketing supervisor for a Chinese game company, a vice president of design for a Chinese game company, and owners of software stores, news kiosks and Internet cafes. We use pseudonyms for all study participants mentioned. We approached players in the wang ba and asked if they would have time for an interview.

\section{Establishing and Maintaining Guanxi}

We observed a wide range of opportunities where a shared gaming context was used to build guanxi. One of the techniques to establish guanxi, for example, was the reciprocal exchange of favors and gifts. Lian, a 50-year old player, told us about her experiences with a fellow player: I chat with people in $Q Q$ games... there was a time that my computer got real slow in accessing the Internet, so I asked a favor from a friend I got to know in $Q Q$ games... he is very nice and he told me he is a computer science major, so I asked him to remotely control my computer and he fixed the problem. In asking another player for computer support, Lian requested a favor, a gesture that rendered the relationship mutual and trustworthy. Even though the two players had never met offline, Lian allowed the other player to remotely control her computer to help her find a solution to the hardware problem. Through the offer of support and the sequential development of expectation to receive future support from Lian if needed, however, a reciprocal exchange took place that strengthened the guanxi that had been based on the shared online gaming context.

Besides playing games together online, physical proximity and shared context outside of the game could provide context for a guanxi base. Many of our study participants felt closer to online friends who lived in the same neighborhood or to in-game friends whom they met regularly at the same physical location, like the Internet cafe or student dormitories. Chenguang, for example, expressed feelings of safety and familiarity to other players who were living in her neighborhood: The ones I feel comfortable meeting offline are the ones that live close to me and we have played together for a long time... Just because we are good online friends doesn't mean we are good friends in real world as well. Another option to build guanxi was to rely on the referral of other players to whom guanxi was already established and maintained, as one of our study participants described: you find out about who is trustworthy from a third party/recommendation by people you know. If you get ripped off, you only get ripped off once. You wouldn't fall for it again.

\subsection{Maintaining Pre-existing Guanxi}

Online gaming in China also supported the maintenance of guanxi that had been developed prior to the game, as was the case for coworkers, couples, parents and children. Ming, for example, a 37-year-old gamer and employee at a publishing house in Chengdu, described that he would sometimes give his female colleagues QQ pets 
(an online casual game): Sometimes, my QQ pets bore baby QQ pets. And, I already have too many $Q Q$ pets [he has $4 Q Q$ pets], so I gave them to my female colleagues as gifts. Interviewer: Why did you give it to them?

Ming: la [literally means to pull, figuratively means to actively build] guanxi. I might need to ask them for help in the future.

Jun and Wei are a young couple in Chengdu. WoW became increasingly important to their guanxi: before WoW, Jun and I hardly had any interests in common. He has his friends and circle, and so do I. I wasn't interested in WoW when Jun first started playing the game. But, then I read some books about the stories behind the game and then started playing and fell in love with it. Now, we have this common hobby. I feel we are more connected. In this example, the online game provided a shared context that allowed the couple to connect in new ways through spending time together online, in the Internet cafe and in their home. Similar to Wei and Jun, we found it common practice that "real life" friends started playing the game together. Extending from their guanxi existent prior to the game, they often established in-game groups and/or guilds that were highly successful and thus prestigious for others to join.

\subsection{Leveraging Pre-existing Guanxi}

At times, players who knew each other prior to the game leveraged their guanxi for in-game profit making. It was, then, often challenging for outsiders to join their activities. Often they also found themselves taken advantage of by the powerful and closely knitted group of guanxi friends. Many players referred to these guild members or player friends as "gold groups," because they would invite others outside the guild to join in-game activities and then take advantage of them. For example: They recruit players from outside the guild to participate in gold raids. At the end of the gold raid, the people from the guild get offline and don't share the gold with the people from outside of the guild. Bing, a software engineer from Beijing commented similarly on his experiences of joining the most prestigious guild on his server: My guild is the best one on the server. Other people might not be able to get some of the equipment, but they succeed at it. But the relationships in the guild are not very good. If you are an outsider looking at this guild, you want to be added, because you see how fast they advance. After you get into this guild, you regret it.

This example illustrates that online gaming did not necessarily always support "good" guanxi. While it was often easy for a group of friends to extend from preestablished guanxi and leverage their guanxi for in-game success, for others these preexisting networks could also lead to the feeling of disconnectedness and exclusion. As in Bing's example, guild structures did not necessarily always provide the familiarity and continuity of interaction that players found so necessary for quality guanxi to develop. Building and maintaining "quality" guanxi, then, usually meant more than just acquiring the right equipment or participating in a stable and prestigious guild. Fen, for example, explained that for guanxi socializing in the game could be more important than equipment: The bad aspect of the guild is that many people join the guild for the equipment and seldom have opportunity for sitting and chatting together, like you would have in real life. This is a change to humanity. 
Most of the players we talked to, however, were able to build new guanxi networks, mainly through leveraging a mix of online and offline resources and connections to others. They developed guanxi with other players in Internet cafes, with other Chinese players on foreign and private servers and when they met others while soloing in the game. Rui, for example, an employee at an Internet cafe in Beijing, told us that he considered some of the players he met in the Internet cafe as part of a trusted circle of friends. Rui moved to Beijing two years ago, which meant leaving friends and family on the one hand and building a new social network on the other. The Internet cafe, a place where he worked, slept and played, provided social and economic infrastructure to gain ground in the new environment.

\section{Developing Quality Guanxi}

Thus far, we have illustrated how online gaming provided the context for a shared guanxi base and have provided insights into the kinds of techniques players deployed to build and maintain guanxi. We will now focus on how dependable and trustworthy relationships were formed in and around online gaming in China, something that players often referred to as "quality" guanxi.

As it happened, our fieldwork took place at a time of considerable turmoil, at least for players of WoW in China. "The Burning Crusade" (TBC), an attractive expansion to WoW in 2007 that introduced among other features the increase in level cap to 70 (60 before), new zones and high-level dungeons, was released on January 16 in Europe, the U.S., and Australia, and on April 20 in regions of Taiwan, Hong Kong, and Macau. In Mainland China, however, TBC was not released until September 2007. Many players found work-arounds to play the TBC. For example, some created accounts on a server outside of Mainland China, e.g. in Taiwan or the U.S. Others accessed unlicensed game servers that provided access to TBC. Players moving to foreign servers or temporarily leaving the game while waiting for the upgrade to be released changed several dynamics in the game. Guilds (in-game groupings of players to help advance in game play) broke apart because of the decreased number of online members. As a result, many WoW players we talked to expressed having difficulty bonding with other online players, because of the increased instability in players' online patterns and the reduced number of players on servers in Mainland China. While these control mechanisms certainly structured the game space, we also found players building trustworthy relationships and quality guanxi in innovative ways, thus finding their own ways around certain restrictions.

[4] observed that "Chinese societies are described as high in particularistic trust, such as among family members, but low in general trust in larger collectives. To the extent that guanxi building represents efforts by individuals to deal with an environment lacking general trust, interpersonal trust is essential in building a quality guanxi." Some of our study participants referred to online gaming environments as lacking general trust. In other words, some players expressed low trust if they were strangers. Others, however, were able to develop trustworthy relationships that often lasted beyond game play. We were, then, intrigued by the question of whether and how online gamers developed interpersonal trust and quality guanxi in and beyond virtual environments. 
Ming, for example, told us that he didn't like to play with strangers in the game and that building trust takes time and resources outside of the game: I usually only play with my work colleagues, but sometimes they have other things to do and cannot play, so I have to play with other people that I don't know. Right after quests, they just grab the loots and run away. I was enraged! Loot should be equally divided. And we usually explicitly agree on that... I hardly trust strangers in games. Lack of trust also occurred in transactions of game resources conducted online. He Peng, a 25 yearold IT consultant in Shanghai, reminisced about an incident of being cheated in WoW: Once I didn't have much time left on my account, so I was in a rush. Somebody next to me [in the game] just happened to sell game point cards and I bought it immediately. The person told me to wait for a minute to activate the card. Later, I learned that he gave the same number to everybody.

In general, low trust in online games was exacerbated by the widespread use of spyware and viruses. Several of our study participants had their game accounts stolen on a PC in an Internet cafe. Many players thus did not trust machines in the Internet cafe. Ming, who we introduced earlier, told us that he bought game objects through face-to-face cash transactions and sometimes both parties needed to log into the game to transfer game objects. Instead of using a computer in the Internet cafe to perform the transaction, he called his wife to log in the game using their home computer.

How did players generally tackle this trust issue and develop quality guanxi? Tao, a 27-year-old gamer in Hangzhou, had played the With Your Destiny game for 4 years and made close friends in the game. He commented: People who seem nice in reality may not be nice online, but people who seem nice online are most likely to be nice in reality as well...[because] it's really hard for someone to pretend [to be nice online] for a long time. During long-term interactions many players started to exchange game accounts and phone numbers as an expression of trust in each other. Tao, whom we introduced in the introduction section, for example, told us how he built strong trust and quality guanxi to other players: We have very good guanxi... We shared our game accounts with each other. When I had already started working, he was still in college. We called each other to talk about games as well as other things. I discussed with him how to prepare in school to find jobs...we played as a highly organized team. We looked after each others' characters... when my account got stolen, my friends immediately offered to buy me a premium account that was worth several thousand RMB (1 Yuan Renminbi is $\sim 0.15$ US\$) at that time. The story of Tao and his close game friends is a telling example of how trust and quality guanxi were built through selfdisclosure and external communication, long-term collaborations, exchange of favors, and a strong sense of comradeship and shared honor. We begin to see then that quality guanxi embodies both deeply emotional values, e.g. Tao's comment “the saddest thing in the game is to see when [our character] was stolen and our enemy bought it", and support in practical manners, e.g. "they offered to buy me a premium account that was worth several thousand RMB..."

\subsection{From Guanxi Base to Quality Guanxi}

Another example of quality guanxi that we found in the context of online gaming was the guanxi between a software shop owner who sells point cards for online games and a gamer who buys point cards from the vendor. Game point cards serve as an economic 
bridge between the real world and game worlds. They convert public currencies into game values. Some of them are time-based, e.g. WoW point cards (30 RMB buys 66 hours of in-game time). Others are in form of game points that can be used to purchase virtual objects and services in the game worlds.

One of our study participants, Lin, ran a software shop that sold game, anti-virus and enterprise software as well as game point cards. Customers could purchase prepaid scratch game cards from Lin. A typical game card came with its sales price in RMB and the value of the corresponding in-game value printed on the front side of the card. After purchase of a game card, customers could then redeem the value of the card into their game accounts using the card number and the password. Alternatively, customers could acquire game point directly from Lin. Lin would then $\log$ in as a vendor into a third party online game card sales system and conduct the purchase for her customer. After the customer paid her back, Lin would often write down the card number and password on a piece of paper and give it to the customer. This option required mutual trust and was thus reserved for Lin's quality guanxi customers: Some of my old customers call me to buy game points. Usually, they transfer money directly to my bank account and I put the game money directly to their game accounts. Since they have been my customers for many years, we are very familiar with each other. I know their game accounts and they know my bank account. We have good guanxi. We are friends and help each other if needed. Once, my shop fell short of cash... I called them and asked them for help. They gave me several thousand RMB...

Lin's example illustrates how interaction through the context of the online game served as a guanxi base for cultivating quality guanxi. If guanxi was to be maintained and turned into quality guanxi as in Lin's case, usually the game-related interactions extended beyond the game, connecting to real-life assets such as the bank account or face-to-face meetings for a transaction.

\subsection{Assessing Guanxi and Interpreting Face}

So far, we have looked at how players built guanxi to engage in trustworthy online and offline interactions and to develop mutual respect for each other. Another aspect that is commonly associated with guanxi in China is mianzi, or face in English. Face refers to one's social status in the eyes of others, which can be accumulated, lost or given to one another and is said to be a key principal guiding relations between Chinese people, rural or urban [9]. While some have speculated about the deterioration of guanxi practices due to industrialization and economic changes $[10,23]$ recent work has provided insight into how guanxi continues to evolve and might even counter-act feelings of anonymity or facelessness $[1,9]$. Hertz, for example, argues that "guanxi locates its practitioners in webs of communitarian contacts and distinguishes them as individuals from the faceless universalized monads who make up the crowd" [9]. Players commented quite frequently on the importance of showing and receiving face through their relationships in and beyond the game. Ju, a student from Beijing, for example, explained how he gained face through his guild, when we asked him what he thought the advantages of being in a guild were: If you can join a strong guild on your server, that is very good... You will feel more face brought by it... For example, your guild is Nol on your server. It is always your guild who wins difficult raids, you will feel honored. When you chat with others, you can mention "I am from..." In the 
game, if players apply to join our guild, they may be refused by the guild, while I can join the top guild, because I have friends in it.

Often, players took specific actions in order to find out about other players' moral attitude and face online. They would, for example, use their secondary characters in order to see others' "true faces", as one player phrased it. Most of the online game players we talked to had several characters distributed across different guilds, sometimes having two or more characters in one guild without revealing to their team members that they are all owned by one player. He Peng explained: Why I have different characters in different guilds? Most players do that. If you have a secondary character, I wouldn't tell others in the guild that it is a secondary character - to protect myself. You use the secondary character to understand what else is going on or to find out about another player's personality and his intentions. If you have really good equipment, people respect you. I use my secondary character to see who still is nice even though I don't have a lot of equipment.

He Peng considered this action a strategy "to protect himself." If a guanxi partner looses face it might also entail loss of face for oneself or one's guild: If people go and do something bad in the name of the guild, for example, they take stuff from the guild which is supposed to be distributed among the members. Others will critique the whole guild for having members like this. The fame of the whole guild is destroyed. Quality guanxi is often associated with a moral and ethical attitude, as well as mutual reliance on each other, which converts into face/status for both guanxi partners when exposed to others $[9,10]$. In confronting a trusted guanxi partner in the guise of a less-advanced character, the moral attitude of the other player could be evaluated.

\section{Discussion}

While guanxi practices in and around online gaming in China provide a telling example of the combination of the instrumental and emotional, it is not something unique to China or online gaming. A series of ethnographic studies in domestic settings, for example, draws attention to the ways in which pragmatic routines of keeping things in order and "configuring" the home bring to the fore symbolic and emotional properties of artifacts and their arrangements in the home $[18,20]$. In a quite different context, we thus found a similar line of reasoning: that through pragmatic means quite emotionally intense outcomes can be generated. In addition, our findings also illustrated how leisure and fun can affect instrumental accomplishment. Bing's example of prestigious but exclusionary guilds brought to the fore how the ability to establish connections to other players outside the in-game structure of the guild or even beyond the game in China's Internet cafes provided opportunity to develop quality guanxi. In contrast, the decision to join a prestigious guild was motivated by the desire to gain reputation and status. Many players established just like Bing a myriad of guanxi connections, offline and online, and hybrids of both, varying in intensity and quality. While in some guanxi networks the emotional aspects prevailed, in others the instrumental aspect came to the fore. However, never did we find a sharp distinction between the instrumental and emotional aspects. Lian built a trustworthy connection to a fellow player and could rely on him for technical support. Chenguang and many others shared game accounts to express trust and mutual reliance, but also to gain knowledge about unfamiliar player 
classes. Similarly, the exchange of QQ pets strengthened the guanxi at stake, while at the same time helping advance the gifted player.

Previous HCI research has highlighted the importance of in-game infrastructures such as guilds and social action around virtual artifacts to support a stable social backdrop to many game activities $[2,5]$. Consistent with this previous work, we found that members of well-run and successful guilds associated feelings of prestige and belonging. But we also came across a range of game practices that fulfilled pragmatic means beyond built-in grouping mechanisms, similar to findings in [15]. For example, despite political decisions such as the delay of the TBC and the pervasive lack of trust in online spaces and Internet cafes, players were able to develop and sustain a myriad of guanxi networks. Here, a pervasive aspect of making the game function for one's own needs was to exploit both digital and physical resources. We have argued elsewhere [11] that a crucial aspect of online gaming in China is the ability to act across an ecology of physical and digital infrastructures and artifacts. The maintenance and development of guanxi flourished exactly because players were able to make use of a mix of digital and physical resources. This evidenced in the cases of Lin and Tao. Lin conducted online transactions, interacted face-to-face, and exchanged bank account information with her customers. Her customers displayed loyalty and trust lending her several thousand RMB when Lin's business underwent a moment of crisis. Similarly, Tao made use of digital and physical resources to build guanxi to his fellow online game players. In exchanging game accounts and phone numbers, he and his online friends expressed trust in one another, eventually allowing for quality guanxi to emerge.

Practices of gold groups (closely knit networks of players who exploit others to gain advantage in the game), on the other hand, evidenced that a functioning social infrastructure in the game is not necessarily always about fair play or mutual support. Rather these groups often functioned so well because of exactly that: demolition of the functioning of others through, for example, exploitation and cheating. Members of gold groups leveraged their pre-existing guanxi networks to form strong and highly collaborative groups in the game. Exploiting both digital interaction and guanxi established outside the game, they not only engaged in highly successful in-game practices, but also strengthened their pre-existing "real life" guanxi. These examples illustrate that functioning in the game meant to artfully interweave online practices with contexts and artifacts that were valued beyond the game. We suggest that if we subscribe a myth of functioning of social groups in online gaming and online communities more broadly that is based on continuity, stability and structure within the online game, we might overlook these other much messier, but quite productive ways of navigating and playing around a regulated game space.

What we take away from these intersections of reciprocal exchange, expression of trust and achievement of instrumental means through game play is an alternate framing of productive and serious gaming. Serious gaming is often associated with the usage of games for educational settings ${ }^{1}$, where through "doing" things in the game, lessons are learned for one's "serious" aspects of life. Thus, serious gaming often implies that gaming can be used as a test bed for the actual "real" that is happening outside of the game. The concept of productive play, on the other hand, is used to

\footnotetext{
${ }^{1}$ See for example: http://www.seriousgames.org
} 
describe work that gets done in the game or relationships that are formed online, which are impacting players' behavior and attitudes outside of the game. Productive play thus has often been used to describe how boundaries between play and work increasingly blur $[3,5,17,22]$. Yee, for example, comments on the irony of online players paying money to game companies for working hard in the game and refers to companies such as IGE that make money from selling virtual currency [22].

What we have found in our research in China is a form of productive play where fun and entertainment were tied to political and social contexts, thus not only producing value within, but accomplishment extrinsic to game play. Online gaming in China was not so much a test bed, as it was an integral part of people's functioning lives. Too often game design for education and work purposes stops with the online space or the assumption of a bounded game space. By expanding our scope and beginning to design entertainment services and games that take into account how people cross and exploit these boundaries, or how they maintain them in order to make games fit their own needs, we begin to leverage the meaningful serious and productive usages of the entertainment space.

\section{Conclusion}

The concept of guanxi arose repeatedly in our encounters with Chinese players of networked games. We might immediately be led to attempt to translate this an apparently equivalent Western understanding of "trust" or perhaps "social capital," but we have deliberately resisted that temptation here. These terms do not capture the way in which guanxi functions and manifests itself as an aspect of everyday social practice for the gamers we worked with. Our concern with "situating" play, then, is not one that attempts to look at the ways that a "Western" game is re-encountered by Chinese players, nor one that attempts to highlight particular issues as somehow essentially Chinese. Rather, we want to draw attention to the ways that the game is encountered and operationalized as a site for the further elaboration of alreadyexisting social relationships and practices. Guanxi does not need to be "implemented" in WoW; for the Chinese players, it is already there as it is in everyday life. In this way, WoW is productive of everyday social relations even as it offers a place of enjoyment and entertainment.

While guanxi is "already" part of WoW, it lives in the margins and the fringes and in the ways WoW is encountered; it is not a designed-in feature of the game. In this way, the creative work-arounds and "productive" ways of play that we have found in the context of online gaming resemble findings from previous research in work-related settings. Studies of organizational work note that organizational structures, rather than prescribing the ways in which work gets accomplished, are the product of orderly work and situated workarounds [7, 12,19]. Similarly, what we mean by "productive" play is not the ability to support stable social groups over time or to define univocally what efficient in-game collaboration might entail. Rather what we refer to are practices of creatively navigating around managed infrastructures, exploiting messy socio-technical settings and leveraging functionalities of a technological system in innovative ways. Guanxi in and around online gaming in China was about cohesive social relations through exchange of trust and favors. Guanxi practice, however, did not necessarily 
lead to the formation of stable groups within the game. Rather, making the game work meant to artfully interweave online practices with contexts and physical artifacts that were valued beyond the game. The "productive" use of the game was not something encoded into the game or planned in advance, rather arose out of the practical contingencies of the actual interaction, both online and offline.

In conclusion, we would like to offer a speculative and provocative outlook. Chinese and U.S. players [14] both rendered Chinese game play at times to be dishonest and lower in status than what was supposedly the case for the West. One might infer that the use of guanxi in game play may be responsible for this, as guanxi might be interpreted as "backward" or "corrupt", something that can be stamped out. One might also suggest that China, characterized as an emerging market, a nation often branded as being in "development", might eventually catch up with the U.S., causing the two game cultures to equalize. The research we presented in this paper, however, casts doubt on such an analysis. Online gaming in China is embedded in a culture that greatly values guanxi and teaches the importance of artful guanxi. The vigorous culture of guanxi tends to strongly intertwine game activities with real life activities. It is here that gaming and the digital and physical sites it engages become arenas for contrived contingency [13]. Considering the growth of social networking technologies in the U.S., China may, instead of lagging behind the U.S., also in certain cases illustrate a precursor of U.S. technology culture in the future.

\section{Acknowledgements}

We would like to thank Bonnie Nardi, Charlotte Lee, Gillian Hayes, Ken Anderson, Thomas Malaby, Lisa Nakamura, Tom Boellstorff, Johanna Brewer and Lilly Irani for their valuable input. We also thank our participants, the Intel Corporation and Intel's People and Practices Research group who supported this work. This work was supported in part by the National Science Foundation under awards 0527729, 0712890,0838601 , and 0838499.

\section{References}

1. Anagnost, A.: National Past-Times. Narrative, Representation, and Power in Modern China. Duke University Press (1997)

2. Brown, B., Bell, M.: CSCW at play: 'There' as a collaborative virtual environment. In: Proc. CSCW 2004, pp. 350-359. ACM Press, New York (2004)

3. Castronova, E.: Synthetic Worlds. The Business and Culture of Online Games. University of Chicago Press (2005)

4. Chen, X., Chen, C.: On the Intricacies of the Chinese Guanxi: A Process Model of Guanxi Development. Asia Pacific Journal of Management 21, 305-324 (2004)

5. Dibbel, J.: Play Money. Or, how I quit my day job and made millions trading virtual loot. Basic Books (2006)

6. Ducheneaut, N., Yee, N., Nickell, E., Moore, R.: The Life and Death of Online Gaming Communities: A Look at Guilds in World of Warcraft. In: Proc. of CHI 2007. ACM Press, New York (2007) 
7. Gerson, E.M., Leigh Star, S.: Analyzing Due Process in the Workplace. ACM Transactions on Office Information Systems 4(3), 257-270 (1986)

8. Gold, T., Guthrie, D., Wank, D.: Social Connections in China. Institutions, Culture and the Changinq Nature of Guanxi. Cambridge University Press, Cambridge (2002)

9. Hertz, E.: Face in the Crowd: The Cultural Construction. In: Chen, N., Clark, C. (eds.) China Urban. Ethnographies of Contemporary Culture. Duke Univ. Press (2001)

10. Kipnis, A.: The Language of Gifts: Managing Guanxi in a North China Village. Modern China 22, 285-314 (1996)

11. Lindtner, S., Nardi, B., Wang, Y., Mainwaring, S., Jing, H., Liang, W.: A Hybrid Cultural Ecology: World of Warcraft in China. In: Proc. of CSCW 2008 (2008)

12. Lutters, W., Ackerman, M.: Beyond Boundary Objects: Collaborative Reuse in Aircraft Technical Support. Computer Supported Cooperative Work 16, 341-372 (2007)

13. Malaby, T.: Beyond Play: A New Approach to Games. Games and Culture 2, 95-113 (2007)

14. Nakamura, L.: Don't Hate the Player, Hate the Game: the Racialization of Labor in World of Warcraft. In: GLS Conference, Madison, Wisconsin (2008)

15. Nardi, B., Harris, J.: Strangers and Friends: Collaborative Play in World of Warcraft. In: Proc. CSCW (2006)

16. Ong, A.: Flexible Citizenship. The Cultural Logics of Transnationality. Duke University Press (1998)

17. Pearce, C.: Productive Play: Game Culture from the Bottom Up. Games and Culture 1(1), 17-24 (2006)

18. Rodden, T., et al.: Between the Dazzle of a New Building and its Eventual Corpse: Assembling the Ubiquitous Home. In: Proc. of Dis 2004, pp. 71-80 (2004)

19. Suchman, L.A.: Office Procedure as Practical Action: Models of Work and System Design. ACM Transactions on Office Information Systems 1(4), 320-328 (1983)

20. Swan, L., Taylor, A., Harper, R.: Making Place for Clutter and Other Ideas of Home. ACM Transactionson Computer-Human Interaction 15(2), Article 9 (2008)

21. Taylor, T.L.: Play Between Worlds: Exploring Online Game Culture. MIT Press, Cambridge (2006)

22. Williams, D., Ducheneaut, N., Xiong, L., Zhang, Y., Yee, N., Nickell, E.: From tree house to barracks: The social life of guilds in World of Warcraft. Games \& Culture 1(4) (2006)

23. Yang, M.: Gifts, Favors and Banquets. The Art of Social Relationships in China. Cornell University Press (1995)

24. Yee, N.: The Labor of Fun: How Video Games Blur the Boundaries of Work and Play. Games and Culture 1, 68 (2006) 